\title{
Carrier Dynamics Resulting from Above and Below Gap Excitation of P3HT and P3HT/ PCBM Investigated by Optical-Pump Terahertz-Probe Spectroscopy ${ }^{\dagger}$
}

\author{
Paul D. Cunningham and L. Michael Hayden* \\ Department of Physics, University of Maryland, Baltimore County, Baltimore, Maryland 21250
}

Received: December 17, 2007; Revised Manuscript Received: February 4, 2008

\begin{abstract}
We report subpicosecond carrier dynamics in photoexcited solid films of regioregular poly(3-hexylthiophene2,5-diyl) (P3HT) and a blend of P3HT/[6,6]-phenyl- $\mathrm{C}_{61}$-butyric acid methyl ester (PCBM) using opticalpump terahertz-probe spectroscopy for excitations well above and below the generally accepted energy gap in P3HT. Both $400 \mathrm{~nm}$ excitation and $800 \mathrm{~nm}$ excitation yield similar results, suggesting the same photoproduct results from these two different excitation energies. The time evolution of the frequency-dependent complex conductivity after optical-pumping reveals characteristics of strong carrier localization. Carrier mobilities and photon-to-carrier yields are estimated based on the application of the Drude-Smith model. Low carrier yields $(\leq 1.5 \%)$ suggest that free carriers are not the primary photogenerated species. High hole mobilities $\left(30-40 \mathrm{~cm}^{2} / \mathrm{Vs}\right.$ ) are consistent with the $\mathrm{THz}$ probing frequency, which selectively probes short-range highly ordered domains. Low-temperature measurements show a slight increase in mobility. Inclusion of PCBM led to an increase in carrier yield for both excitation wavelengths.
\end{abstract}

\section{Introduction}

Semiconducting polymers have attracted much interest for potential applications in organic opto-electronic devices such as organic field-effect transistors (OFET), organic light-emitting diodes (OLED), and organic photovoltaics (OPV). Regioregular poly(3-hexylthiophene) (RR-P3HT) has been widely studied, as reports of high field-effect mobilities ${ }^{1}$ show promise for such devices. Field-effect hole mobilities ${ }^{2}$ as high as $0.2 \mathrm{~cm}^{2} / \mathrm{Vs}$, approaching the mobility of amorphous silicon, have been reported. The small bandgap ${ }^{3-6}(1.9 \mathrm{eV} / 653 \mathrm{~nm})$ and corresponding absorption overlaps well with the strongest spectral components of incident sunlight. The highest efficiencies achieved in OPVs ${ }^{7}$ have been based on a P3HT and $[6,6]-$ phenyl- $\mathrm{C}_{61}$-butyric acid methyl ester (PCBM) bulk heterojunction. Many factors influencing device performance, such as the molecular weight of $\mathrm{P} 3 \mathrm{HT},{ }^{8}$ blend composition,,${ }^{9,10}$ annealing,,${ }^{11-13}$ and film deposition, ${ }^{10,14-16}$ have been examined. These studies do not all agree and report widely varying mobility values $\left(10^{-5}-10^{-1} \mathrm{~cm}^{2} / \mathrm{Vs}\right)$. The common thread in these studies is that they indirectly probe charge carrier dynamics. Device constraints present in OFET, time-of-flight (TOF), and photoconductivity (PC) measurements, lead to complications by electrical contacts, high electric fields, and the need for carriers to travel long distances to reach the electrodes. ${ }^{17,18}$ All-optical techniques can avoid many of these complications, but some all-optical techniques such as photoinduced absorption and photoluminescence spectroscopy are inherently indirect methods where charge carrier dynamics are inferred from spectral signatures of electronic transitions. ${ }^{19,20}$ Optical-pump terahertzprobe (OPTP) (i.e., time-resolved terahertz spectroscopy) offers the additional advantage of being directly sensitive to charge carriers. $^{21}$ Time-resolved microwave conductivity (TRMC) experiments, which have also been applied to the study of semiconducting polymers such as polyphenylene vinylene (PPV) and $\mathrm{P} 3 \mathrm{HT},{ }^{4,22,23}$ are sensitive to charge carriers but are typically

\footnotetext{
$\dagger$ Part of the "Larry Dalton Festschrift".

* Corresponding author. E-mail: hayden@umbc.edu.
}

limited to nanosecond resolution. ${ }^{21}$ OPTP provides subpicosecond resolution of carrier dynamics with the additional benefit of a coherent measurement technique, providing access to the real and imaginary parts of the frequency-dependent complex conductivity. ${ }^{24,25}$ This means that OPTP has the unique ability to differentiate between free and bound excitations. ${ }^{21,26}$ However OPTP, like TRMC measurements, is not able to distinguish between the electron and hole contributions to the mobility and yields their sum. This is not a particular problem for polymer systems such as P3HT where the hole mobility is thought to be several orders of magnitude larger than the electron mobility. ${ }^{1}$

In recent years, OPTP has been used to study MEH-PPV, ${ }^{19,27}$ pentacene, ${ }^{28,29}$ functionalized pentacene, ${ }^{30,31}$ rubrene, ${ }^{32,33}$ P3HT, ${ }^{26}$ and its bulk heterojunction blend with PCBM. ${ }^{21}$ Two previous terahertz studies on $\mathrm{P} 3 \mathrm{HT}$ have been performed. These studies, by Hendry et al. ${ }^{26}$ and Ai et al., ${ }^{21}$ examining the photoconductive properties of P3HT, differ in their predicted carrier yields by an order of magnitude. The former does not report a mobility and employs a scaled model of the frequencydependent mobility of isolated polymer chains in solution ${ }^{34}$ to extract carrier yields. Both of these studies were performed by exciting P3HT well above the band edge.

Up to this point, no study has examined the photoconductivity in these materials when exciting them below the generally accepted band gap of $1.9 \mathrm{eV}$. In this paper, we examine charge carrier generation and dynamics in P3HT and a 1:1 blend of $\mathrm{P} 3 \mathrm{HT} / \mathrm{PCBM}$ when excited below the band edge $(1.55 \mathrm{eV} / 800$ $\mathrm{nm})$ and well above the gap $(3.1 \mathrm{eV} / 400 \mathrm{~nm})$ and show that the photoresponses are similar. This suggests the existence of one type of photoproduct regardless of the excitation wavelength. Our results confirm the low photon-to-carrier yields in these polymer systems as seen by others. ${ }^{19,26}$ In addition, we present the first low-temperature terahertz conductivity measurements of these material systems. We find negligible dependence of the photoconductivity on temperature. 


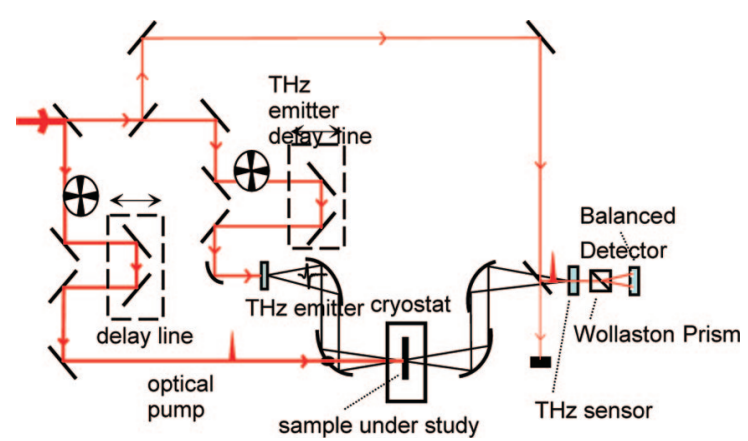

Figure 1. Schematic diagram of OPTP spectroscopy setup. A reference scan of the unexcited sample is collected while mechanically chopping the terahertz emitter beam, and pump/probe difference scans are collected while mechanically chopping the optical pump beam.

\section{Experimental Methods}

Samples were prepared in air from RR-P3HT (Aldrich, molecular weight $87 \mathrm{~kg} / \mathrm{mol},>98.5 \% \mathrm{RR}$ ) and the soluble fullerene derivative PCBM (Nano-C). Pure RR-P3HT and $\mathrm{P} 3 \mathrm{HT} / \mathrm{PCBM}$ 1:1 weight fraction solutions were prepared. We chose to examine, exclusively, the $1: 1$ blend, as it has been shown to have the best performance. ${ }^{9,21}$ Thin samples $(<2 \mu \mathrm{m})$ were spun cast from either chloroform or chlorobenzene solutions onto grade V1 muscovite mica (Tedpella, Inc.) substrates. The solution concentration was varied to control the thickness of the films. Thicker samples $(3-40 \mu \mathrm{m})$ with optical density $(\mathrm{OD})>0.4$ at $800 \mathrm{~nm}$ were drop cast. All films were allowed to dry in air while covered by a Petri dish, as this has been shown to result in well-ordered films. ${ }^{15}$ For OPTP studies, only films with $\mathrm{OD}>2$ at both pump wavelengths were used.

We performed linear ultraviolet and visible absorption spectroscopy using a Perkin-Elmer Lambda 3B spectrophotometer. The absorption coefficient was found from the absorbance spectra using $\alpha=\mathrm{OD} \ln (10) / z$, where $z$ is the thickness. Film thicknesses were determined using a KLA Tencor Alpha-Step IQ surface profilometer.

The setup of our OPTP experiment is similar to that reported by others ${ }^{24,35}$ (Figure 1). The fundamental laser source for terahertz generation, detection, and optical excitation is a 700 $\mu \mathrm{J} 1 \mathrm{kHz}$ repetition rate, $50 \mathrm{fs} 800 \mathrm{~nm}$ amplified Ti:Sapphire laser (Spectra-Physics, Spitfire). We use a Au-coated $90^{\circ}$ offaxis parabolic (OAP) mirror to focus $270 \mu \mathrm{J}$ to $2.8 \mathrm{~mm}$ onto a $1 \mathrm{~mm}$ thick $\langle 110\rangle \mathrm{ZnTe}$ crystal where the THz probe is generated via optical rectification. ${ }^{36}$ The $\mathrm{THz}$ radiation is collected, collimated, and focused to $<1 \mathrm{~mm}$ onto the sample using a standard 4F arrangement of 4" OAP mirrors. The transmitted terahertz radiation is collected and refocused onto a $2 \mathrm{~mm}$ thick $\langle 110\rangle \mathrm{ZnTe}$ crystal using another 4F arrangement of OAP mirrors. A small part of the fundamental $(700 \mathrm{~nJ})$ is focused onto the ZnTe detector crystal, where collinear propagation with the terahertz beam is achieved using a silicon wafer beam combiner. The terahertz waveform is detected using free-space electro-optic sampling, ${ }^{36,37}$ where the gating beam is focused onto unbiased balanced silicon photodiodes. It is important to point out that, through this coherent detection scheme, the terahertz electric field is sampled. For the $800 \mathrm{~nm}$ pump (excitation) beam, the fundamental is attenuated, copropagated with the terahertz beam via a hole in an OAP mirror and focused to $2.3 \mathrm{~mm}$ onto the sample. We obtain the $400 \mathrm{~nm}$ pump beam by frequency doubling the $800 \mathrm{~nm}$ pump beam in a $1 \mathrm{~mm}$ thick $\beta$-barium borate crystal, resulting in a $2.1 \mathrm{~mm}$ focus at the sample. It is important to ensure that the pump beam is at least twice as large as the terahertz beam at the sample so that a

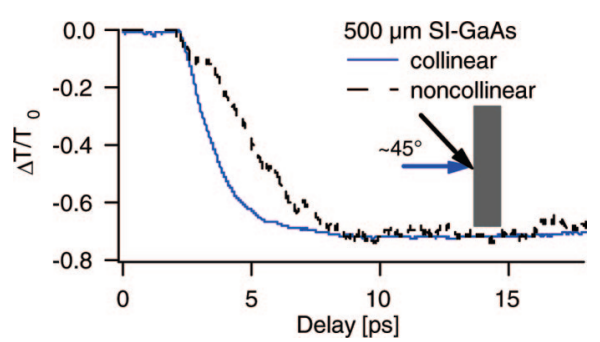

Figure 2. A comparison of $800 \mathrm{~nm}$ collinear and noncollinear pumping during OPTP spectroscopy. The point at which the signal levels off has been displaced from $\sim 5.5 \mathrm{ps}$ to $\sim 7.8 \mathrm{ps}$, which is consistent with the $45^{\circ}$ angle of incidence.
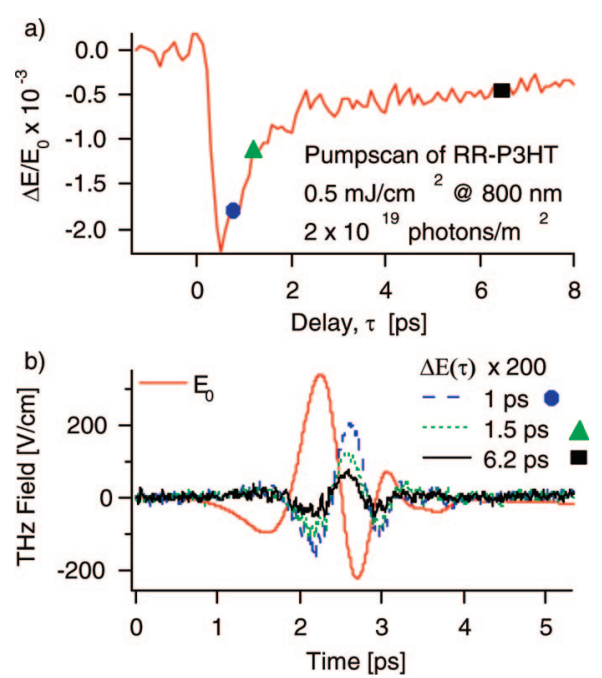

Figure 3. Typical (a) pump and (b) probe scans acquired from RRP3HT. The probe scans superimposed on a reference scan of the unexcited material have been multiplied by a scaling factor 200 and correspond to the delays indicated (closed symbols) on the pump scan.

uniformly excited area is probed. ${ }^{35}$ Also, note that pumping is done collinearly to eliminate temporal smearing introduced by noncollinear geometries ${ }^{24}$ (Figure 2). For low-temperature measurements, the sample was housed in an Oxford Instruments liquid He optical cryostat.

There are two modes of operation for OPTP experiments, pump and probe scans. In pump scans, the peak of the transmitted $\mathrm{THz}$ waveform is monitored while the pump delay line is scanned backward, decreasing the optical path length traveled by the pump beam and pushing the arrival of the pump pulse at the sample to earlier times, creating a positive delay for the terahertz probe beam. At a delay of zero, the pump and probe beams are temporally overlapped within the sample. For positive terahertz probe beam delays, there is a reduction in the transmitted terahertz beam due to photoexcitation of the sample. The pump beam is mechanically chopped, and a lockin amplifier is used to monitor the pump-induced changes in the terahertz transmission, $\Delta E(t, \tau)$, where $\tau$ is the delay between the arrival of the pump and probe beams, and $t$ is the time domain variable of the terahertz pulse. This chopping arrangement is essential to detect the small changes in terahertz transmission when examining thin photoconductive polymer films. By adjusting the delay between the pump and probe beam, the evolution of $\Delta E / E_{0}(t, \tau)$, i.e., the average dynamics of the excited state, are obtained (Figure $3 \mathrm{a}$ ). When very little phase shift is present in $\Delta E(t, \tau)$, the pump scan $\Delta E / E_{0}(t, \tau)$ is proportional to the product of the carrier density, $N(\tau)$, and the mobility $\mu(\tau)$. If a significant phase shift exists, the observed 


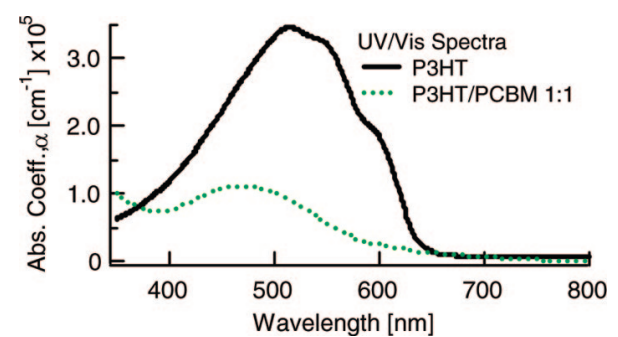

Figure 4. Representative UV-visible absorption spectra of P3HT and P3HT/PCBM 1:1. Absorption depths reported in the text are average values from all films examined.

change in terahertz field amplitude is not simply related to the conductivity, and the pump scan does not yield useful dynamics. For probe scans, the complete terahertz waveform is obtained at a fixed pump delay by scanning the terahertz emitter delay line backward, decreasing the optical path traveled by the terahertz pulse allowing for EO sampling from the leading to the trailing edge of the terahertz waveform (Figure 3b). Keeping the arrival times of the pump pulse at the sample and the sampling pulse at the terahertz sensor fixed ensures that each point on the sampled terahertz waveform experiences the same delay and thus probes the same excited-state of the sample. ${ }^{38}$ Through Fourier analysis, the frequency-dependent complex conductivity is obtained allowing for modeling of the response and extraction of the carrier density and mobility. Following the work of Nienhuys and Sundstrum, ${ }^{27,39}$ we use the following analytic approximation to extract the frequency dependent complex conductivity from our probe scans:

$$
\tilde{\sigma}(\omega, \tau) \approx-\frac{n_{\mathrm{THz}}+n_{\mathrm{air}}}{Z_{0} d} \frac{\Delta \tilde{E}(\omega, \tau)}{\tilde{E}_{0}(\omega, \tau)}
$$

where $Z_{0}$ is the impedance of free space, $d$ is the (1/e) absorption depth of the pump beam, and $n_{\mathrm{THz}}$ and $n_{\text {air }}$ are the terahertz indices of refraction for the unexcited sample and air $(n=1)$, respectively. For many polymers, there is very little absorption, and we can take the terahertz index of refraction to be frequency independent. The terahertz indices $\left(n_{\mathrm{THz}}\right)$ of P3HT and P3HT/ PCBM 1:1 are known to be 2.22 and 1.79, respectively. ${ }^{21}$ It is important to note that the analytic approximation in eq 1 only holds for small $\Delta E / E_{0}(t, \tau)$ and ignores multiple reflections within the sample. However, it has been observed that more general extraction techniques yield similar results within this limit. ${ }^{21}$

\section{Results/Discussion}

Figure 4 shows linear absorption spectra of P3HT and P3HT/ PCBM 1:1 films. In RR-P3HT, the first polaronic absorption is reported as being in the range $1.74-2.14 \mathrm{eV}(713-579 \mathrm{~nm}){ }^{5}$ However, there is also nonzero absorption further into the infrared. We determined absorption depths of $170 \pm 30 \mathrm{~nm}$ at a wavelength of $400 \mathrm{~nm}$ and $3 \pm 1 \mu \mathrm{m}$ at $800 \mathrm{~nm}$ by measuring the absorbance in 20 films of varying thicknesses $(100-40 \mu \mathrm{m})$ and averaging those results. The three peaks present in the P3HT spectrum at 596, 547, and $514 \mathrm{~nm}$ correspond to the three bands of the $\pi-\pi^{*}$ transition. ${ }^{3}$ As the shoulder at $596 \mathrm{~nm}$ is not present in the published spectra of P3HT in solution, it is typically attributed to interchain interactions. ${ }^{13,40}$ The absorption tail extending into the infrared may be the $(1.35 \mathrm{eV} / 919 \mathrm{~nm})$ triplet state previously seen in the electroluminescence spectra of polythiophenes. ${ }^{41}$ Here the presence of the heavy sulfur atom could provide the necessary spin-orbit coupling to weakly allow this spin-forbidden transition. Doping the polymer with PCBM
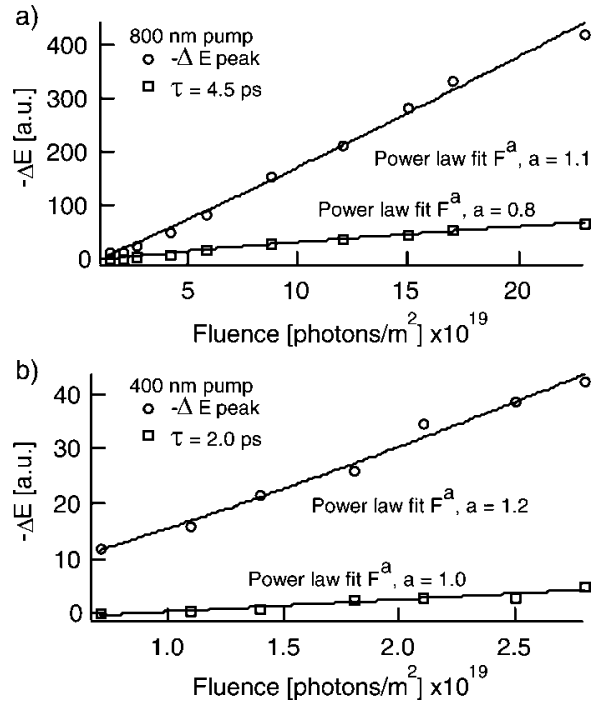

Figure 5. Pump-fluence dependence of the change in terahertz transmission for (a) $800 \mathrm{~nm}$ excitation taken at the pump signal peak (circles) and at a delay of $4.5 \mathrm{ps}$ (squares) and for (b) $400 \mathrm{~nm}$ excitation taken at the pump signal peak (circles) and at a delay of $2.0 \mathrm{ps}$ (squares). The lines are power-law fits to the data.

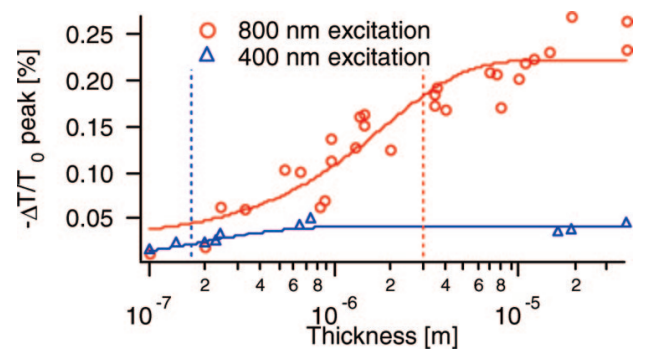

Figure 6. Thickness dependence of the peak change in terahertz transmission observed during pump scans for an $800 \mathrm{~nm}$ pump (circles) and a $400 \mathrm{~nm}$ pump (triangles). The solid lines are exponential fits to the data from which estimates of the absorption depths are found. Absorption depths determined by UV/visible spectroscopy are noted (vertical lines).

leads to beaching of the $\pi-\pi^{*}$ absorption. These spectral alterations have been attributed to decreased interchain interaction of the polymer and the presence of electron-transfer to the fullerene derivative. ${ }^{3}$ The shoulder near $600 \mathrm{~nm}$, seen in wellordered thin films of P3HT but not present in published spectra from solution and disordered films, ${ }^{21}$ implies there is some retention of two-dimensional lamellar ordering of the $\mathrm{P} 3 \mathrm{HT}$ molecules in the bulk heterojunction. We measured the absorption depths of the P3HT/PCBM 1:1 blend at 400 and $800 \mathrm{~nm}$ to be $240 \mathrm{~nm}$ and $5 \mu \mathrm{m}$, respectively.

In Figure 5, the initial photoresponse ( $-\Delta E$ peak) exhibits a linear dependence on pump fluence for both 400 and $800 \mathrm{~nm}$ excitation. We conclude that carrier generation at $400 \mathrm{~nm}$ (Figure 5b) is the result of a linear process and not the dissociation of hot excitons via sequential absorption. ${ }^{42}$ On the basis of the observed linear pump-fluence dependence, we are confident that exciton-exciton annihilation, ${ }^{4}$ previously observed in nanosecond-resolution TRMC studies for fluences of $<10^{18} \mathrm{~m}^{-2}$ and resulting in sublinear fluence dependence for excitation between 1.91 and $2.88 \mathrm{eV}(649-431 \mathrm{~nm})$, does not influence the dynamics on subpicosecond time scales. The linear fluence dependence at $800 \mathrm{~nm}$ indicates that the terahertz response is not due to a second-order process such as two-photon absorption, which has only been observed ${ }^{41}$ in polythiophenes at excitation energies of $>2.2 \mathrm{eV}$ (i.e., wavelengths of $<564$ 

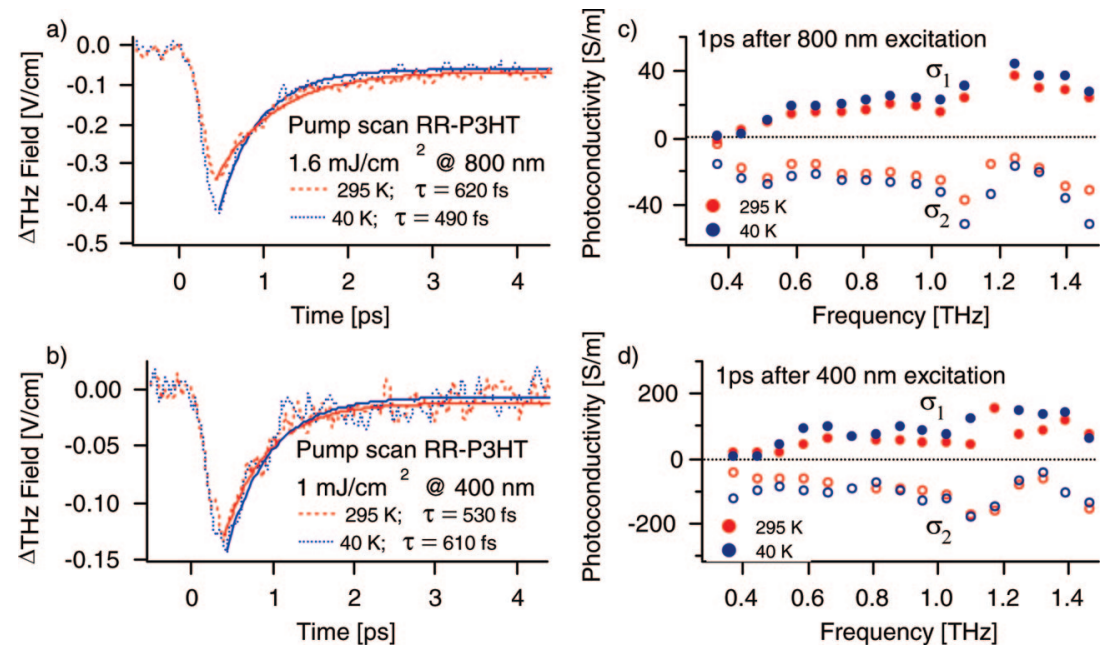

Figure 7. Pump scans performed using (a) $800 \mathrm{~nm}$ and (b) $400 \mathrm{~nm}$ pumps at both $40 \mathrm{~K}$ and room temperature. Lines are stretched exponential fits to the data with a time constant $\tau$. The real (closed circle) and imaginary (open circle) photoinduced conductivity of RR-P3HT for (c) $800 \mathrm{~nm}$ and (d) $400 \mathrm{~nm}$ excitation is extracted at $40 \mathrm{~K}$ and room temperature for a delay of $1 \mathrm{ps}$. The resonance at $1.1 \mathrm{THz}$ is caused by absorption in water vapor, as inclusion of a cryostat prohibited purging the entire terahertz beam path with dry air.
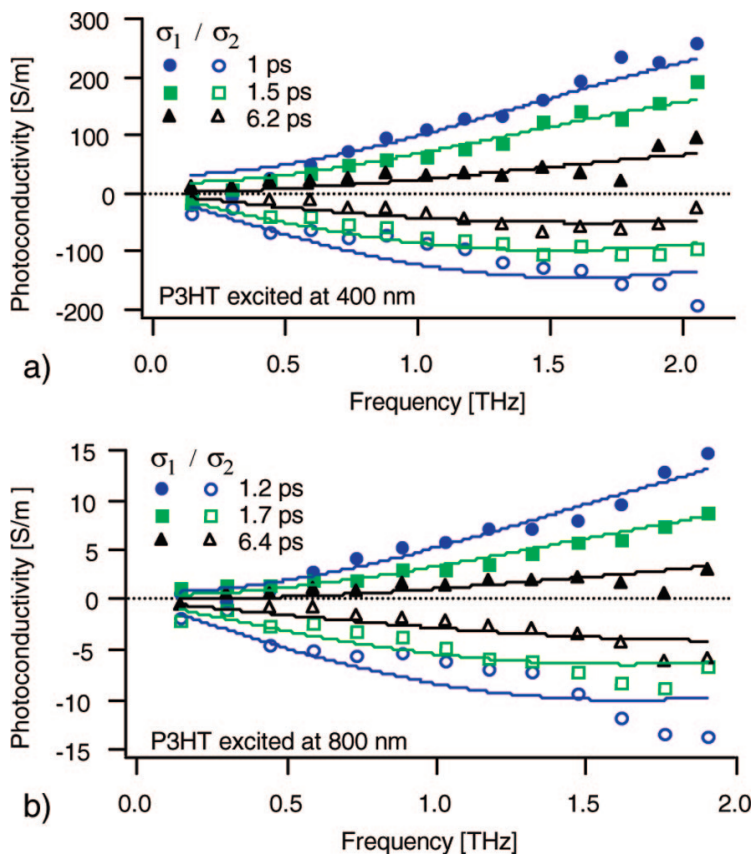

Figure 8. Extracted complex conductivity of RR-P3HT measured at $1 \mathrm{ps}$ (circles), $1.5 \mathrm{ps}$ (squares), and $6.2 \mathrm{ps}$ (triangles) for (a) $400 \mathrm{~nm}$ excitation, and $1.2 \mathrm{ps}$ (circles), $1.7 \mathrm{ps}$ (squares), and $6.4 \mathrm{ps}$ (triangles) for (b) $800 \mathrm{~nm}$ excitation at a constant fluence of $2 \times 10^{19}$ photons/ $\mathrm{m}^{2}$. Lines are fits to the data using the DS model; the fitting results are displayed in Table 1 and discussed in the text.

$\mathrm{nm})$. The sublinear photoinduced absorption with fluence seen at long delays is indicative of second-order carrier-carrier recombination processes.

Figure 6 shows that the photoresponse at $800 \mathrm{~nm}$ excitation increases up to the absorption depth in the material, at which point it begins to level off in accordance with a Beer-Lambert dependence of the absorption on film thickness. We see the same behavior for $400 \mathrm{~nm}$ excitation, where the short absorption depth leads to a constant response for films thicker than $800 \mathrm{~nm}$. Exponential fits to $y=y_{0}-A \mathrm{e}^{- \text {Thickness*d }}$ yield absorption depths (d) similar to those obtained from UV/visible spectroscopy, 2 $\pm 0.5 \mu \mathrm{m}$ and $200 \pm 100 \mathrm{~nm}$ for 800 and $400 \mathrm{~nm}$ excitation, respectively.

Figure 7a,b compares the photoresponse at $40 \mathrm{~K}$ to that at room temperature for both pump wavelengths. The detected
TABLE 1: Results of DS Model Fits to the Extracted Frequency-Dependent Complex Conductivity of RR-P3HT in Figure 8

\begin{tabular}{|c|c|c|c|c|c|c|}
\hline $\begin{array}{c}\text { delay } \\
\text { (ps) }\end{array}$ & $\tau(\mathrm{fs})$ & $\begin{array}{c}\mu \\
\left(\mathrm{cm}^{2} / \mathrm{Vs}\right)\end{array}$ & $\begin{array}{c}N \\
\left(\times 10^{22} \mathrm{~m}^{-3}\right)\end{array}$ & $\varphi(\%)$ & $c$ & $\begin{array}{c}\mu_{\mathrm{DC}} \\
\left(\mathrm{cm}^{2} / \mathrm{Vs}\right)\end{array}$ \\
\hline \multicolumn{7}{|c|}{ P3HT: $400 \mathrm{~nm}$ Excitation } \\
\hline 1.0 & $40 \pm 4$ & $42 \pm 4$ & $100 \pm 20$ & $0.8 \pm 0.3$ & -0.95 & $2.1 \pm 0.9$ \\
\hline 1.5 & $43 \pm 3$ & $44 \pm 3$ & $61 \pm 8$ & 0.5 & -0.96 & $1.8 \pm 0.5$ \\
\hline 6.2 & 40 & 41 & $31 \pm 2$ & $0.3 \pm 0.1$ & -0.99 & $0.4 \pm 0.8$ \\
\hline \multicolumn{7}{|c|}{ P3HT: $800 \mathrm{~nm}$ Excitation } \\
\hline 1.2 & $33 \pm 4$ & $34 \pm 4$ & $9 \pm 0.9$ & $1.5 \pm 0.7$ & -0.97 & $1.0=$ \\
\hline 1.7 & $30 \pm 3$ & $31 \pm 3$ & $6.7 \pm 0$ & $1.1 \pm 0.5$ & -0.96 & $1.2 \pm 0.3$ \\
\hline 6.4 & 30 & 31 & $3.4 \pm 0.2$ & $0.6 \pm 0.2$ & -1 & $0.0 \pm 0.6$ \\
\hline
\end{tabular}

terahertz field is calculated using the relation derived by Planken et al. ${ }^{43}$ Contrary to previous reports, ${ }^{20,44}$ we observe negligible temperature dependence of the long-lived photoconductivity over this broad temperature range. This is inconsistent with an activated hopping model for conduction. ${ }^{5}$ The dark lines are stretched exponential fits to the data, where the stretching parameter is related to the width of the distribution of carrier recombination rates. We see no strong dependence of the stretching parameter on temperature. However, the magnitude of the peak change in transmission increases with decreased temperature. Figure $7 \mathrm{c}$,d shows that the frequency dependence of the photoconductivity does not change significantly with temperature. Since the pump fluence is held constant, we assume the carrier density remains the same and attribute the slight increase in photoconductivity to an increase in the mobility with decreased temperature. This temperature dependence is characteristic of metallic (i.e., band-like) behavior in organic crystals. $^{28,31,45}$ In those systems, as the temperature decreases, so does the number of phonons, leading to an increase in scattering time and corresponding increase in the mobility. However, this is unexpected for a polymer system where there is no lattice to provide the means for thermally activated scattering. A decrease in mobility with increased temperature has been seen in isolated chains of $\mathrm{PPV}^{19}$ and attributed to increased torsion disorder, decreasing interchain interactions. In thin films of P3HT, charge modulation spectroscopy ${ }^{40}$ has revealed a similar increase in torsion disorder with temperature, which may lead to the reduced mobility seen in our results. 

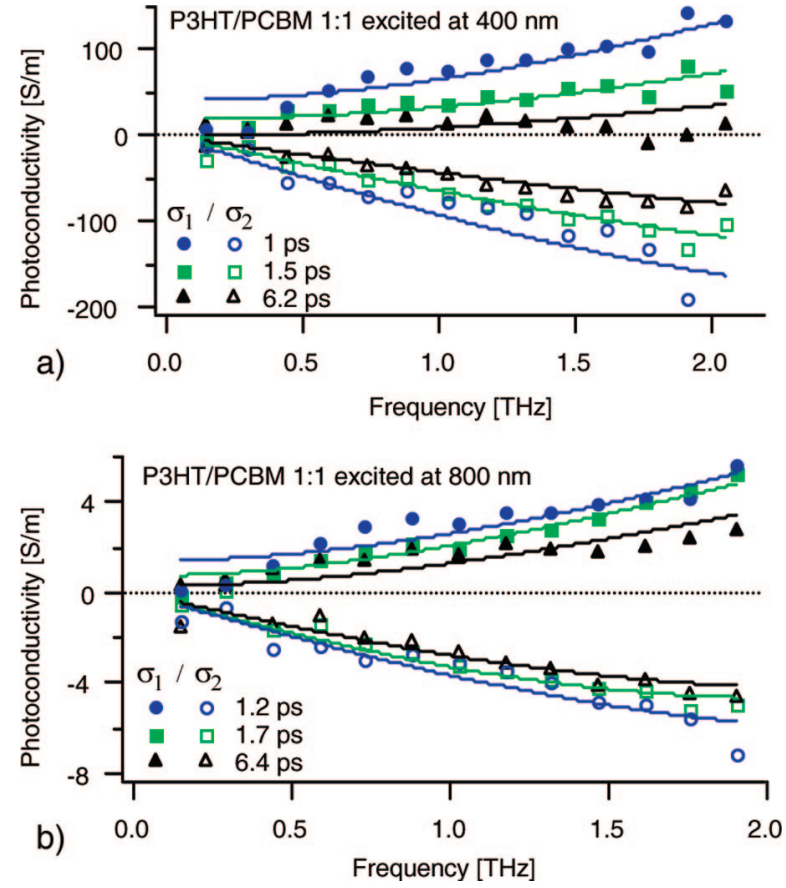

Figure 9. Extracted complex conductivity of P3HT/PCBM 1:1 measured at $1 \mathrm{ps}$ (circles), $1.5 \mathrm{ps}$ (squares), and $6.2 \mathrm{ps}$ (triangles) for (a) $400 \mathrm{~nm}$ excitation, and $1.2 \mathrm{ps}$ (circles), $1.7 \mathrm{ps}$ (squares), and $6.4 \mathrm{ps}$ (triangles) for (b) $800 \mathrm{~nm}$ excitation at a constant fluence of $2 \times 10^{19}$ photons $/ \mathrm{m}^{2}$. Lines are fits to the data using the DS model; the fitting results are displayed in Table 2.

TABLE 2: Results of DS Model Fits to the Extracted Frequency-Dependent Complex Conductivity of P3HT/ PCBM 1:1 in Figure 9

\begin{tabular}{|c|c|c|c|c|c|c|}
\hline $\begin{array}{l}\text { delay } \\
\text { (ps) }\end{array}$ & $\tau(\mathrm{fs})$ & $\begin{array}{c}\mu \\
\left(\mathrm{cm}^{2} / \mathrm{Vs}\right)\end{array}$ & $\begin{array}{c}N \\
\left(\times 10^{22} \mathrm{~m}^{-3}\right)\end{array}$ & $\varphi(\%)$ & $c$ & $\begin{array}{c}\mu_{\mathrm{DC}} \\
\left(\mathrm{cm}^{2} / \mathrm{Vs}\right)\end{array}$ \\
\hline \multicolumn{7}{|c|}{ P3HT/PCBM 1:1: $400 \mathrm{~nm}$ Excitation } \\
\hline 1.0 & $20 \pm 4$ & $21 \pm 4$ & $260 \pm 90$ & $3 \pm 1$ & -0.952 & $1 \pm 0.3$ \\
\hline 1.5 & $17 \pm 3$ & $18 \pm 3$ & $230 \pm 70$ & $3 \pm 1$ & -0.971 & $0.5 \pm 0.1$ \\
\hline 6.2 & 17 & 18 & $150 \pm 10$ & $1.9 \pm 0.3$ & -1 & $0.0 \pm 0.2$ \\
\hline \multicolumn{7}{|c|}{ P3HT/PCBM 1:1: $800 \mathrm{~nm}$ Excitation } \\
\hline 1.2 & $25 \pm 4$ & $26 \pm 4$ & $7 \pm 2$ & $1.9 \pm 0.7$ & -0.95 & $1.3 \pm 0.3$ \\
\hline 1.7 & $31 \pm 3$ & $32 \pm 3$ & $4.0 \pm 0.6$ & $1.3 \pm 0.6$ & -0.964 & $1.2 \pm 0.3$ \\
\hline 6.4 & 28 & 29 & $3.8 \pm 0.2$ & $1.0 \pm 0.2$ & -0.984 & $0.5 \pm 0.3$ \\
\hline
\end{tabular}

The extracted conductivity for the $400 \mathrm{~nm}$ pump is very similar to those reported elsewhere ${ }^{21,26}$ (Figure 8 ). The observed frequency dependence, negative $\operatorname{Im}[\sigma(\omega)]$ with both real and imaginary components increasing in magnitude with frequency, is characteristic of dispersive transport in a disordered medium with inhibited long-range transport. ${ }^{19,21,26,27,34,46}$ The real conductivity exhibits a power law frequency trend with exponent $1<n<2$ that is universally observed for polymers. ${ }^{46,47}$ The real component of the photoconductivity decays with delay, which agrees with other reports. ${ }^{21}$ The conductivity exhibits the same frequency-dependent trend for both excitation wavelengths, where the order of magnitude lower conductivity for the 800 $\mathrm{nm}$ pump is due to the longer absorption depth at $800 \mathrm{~nm}$ and the associated lower carrier density.

Materials with nanoscale disorder, such as polymers and nanocrystalline materials, exhibit strong carrier localization, where random defects cause enough destructive interference among scattering events to slow carrier propagation. This carrier localization can be described by the Drude-Smith (DS) model. In his work on liquid $\mathrm{Hg}, \mathrm{Smith}^{48}$ proposed the following generalization of the well-known Drude model:

$$
\tilde{\sigma}(\omega)=\frac{N e^{2} \tau}{m^{*}(1-i \tau \omega)}\left(1+\sum_{n=1}^{\infty} \frac{c_{n}}{(1-i \tau \omega)^{n}}\right)
$$

where $N$ is the carrier density, $\tau$ is the scattering time, $m^{*}$ is the effective mass, and $c$ is the persistence of velocity of each scattering event. Typically, the series is truncated, keeping only $c_{1}$ to describe the first scattering event. If $c_{1}=0$, we regain the Drude expression, whereas $-1<c_{1}<0$ describes varying degrees of back-scattering that are characteristic of localized carriers. Correspondingly, when $c_{1}<0$, the DC conductivity is suppressed and the oscillator strength becomes shifted to higher frequencies. ${ }^{48}$ An estimate of the DC conductivity is given by $\tilde{\sigma}(0)=e N \mu\left(1+c_{1}\right)$. It is worth mentioning that the DS model can accommodate materials near the metal-insulator transition, ${ }^{49}$ where the localization-modified Drude model cannot. ${ }^{21}$

To apply the DS model, we have truncated the series in eq 2 and separated it into its real and imaginary parts. We perform a global fit procedure to determine the best fit of the DS model to the extracted complex conductivity. Here the real part is fit to $\sigma_{1}(\omega)$, and the imaginary part is fit to $\sigma_{2}(\omega)$ simultaneously, while the effective mass ${ }^{50}$ is held constant at $1.7 m_{e}$, yielding the scattering time, carrier density, and persistence of velocity. Note that any uncertainty in the effective mass translates directly to uncertainty in the extracted carrier density as well as calculated mobility through $\mu=e \tau / m^{*}$. We calculate the photonto-carrier yields from the extracted carrier density given the known fluence, corrected for reflective losses using estimates of the visible indices of refraction, ${ }^{51}$ and absorption depth using $\phi=n d(\lambda) / F T(\lambda)$, where $F$ is the fluence, $d(\lambda)$ is the absorption depth, and $T(\lambda)$ is the Fresnel transmissivity, which are both functions of the pump wavelength $\lambda$.

The mobilities found using the DS model can be found in Table 1. These values are several orders of magnitude larger than those reported for OFETs $s^{1,2}$ or in the microwave regime..$^{4,20}$ A comparison between the mobility found by all optical techniques versus those that use electrical contacts (e.g., OFET, TOF, PC) is difficult, as the carriers must travel the length of the device $(\sim 100 \mathrm{~nm})$ to reach the contacts and are subject to potential barriers at the contacts, ${ }^{17}$ grain boundaries, conjugation breaks, large interunit angles, gate-induced doping, and high electric fields. ${ }^{18,52}$ The high frequency, low field $(<1 \mathrm{kV} / \mathrm{cm})$ probe used in OPTP experiments measures the carrier mobility over short highly ordered domains. ${ }^{18,23}$ It has been shown that increasing the probing frequency in the gigahertz range by a factor of 3 can yield nearly an order of magnitude increase in the extracted mobility ${ }^{53}$ due to the shorter mean square distances traveled by carriers during the shorter period oscillation. So we would expect the even higher frequency terahertz probe to yield a correspondingly larger extracted mobility. It should be pointed out that, though these difficulties are encountered for polymers and disordered media, terahertz mobilities found for bulk inorganic semiconductors and organic crystals are in good agreement with those found through other techniques. ${ }^{29,30,35,54}$

For a meaningful comparison of the results shown here, we must look at all-optical techniques sensitive to conductivity, such as TRMC. P3HT has a mobility at $9 \mathrm{GHz}^{4}$ of $0.014 \mathrm{~cm}^{2} / \mathrm{Vs}$. If we scale this value to the terahertz regime, assuming a $\omega^{1.6}$ scaling factor as seen in other polythiophenes, ${ }^{55}$ we expect a mobility of $26 \mathrm{~cm}^{2} / \mathrm{Vs}$, which is similar to our values. The mobility extracted at $800 \mathrm{~nm}$ excitation is similar to those found for $400 \mathrm{~nm}$ excitation. This may be evidence that both excitations ultimately yield the same photoproduct in P3HT. The photon-to-carrier yields $(\leq 1.5 \%)$ are in agreement with other reports. ${ }^{4,26}$ Such a low carrier yield suggests that free 
a
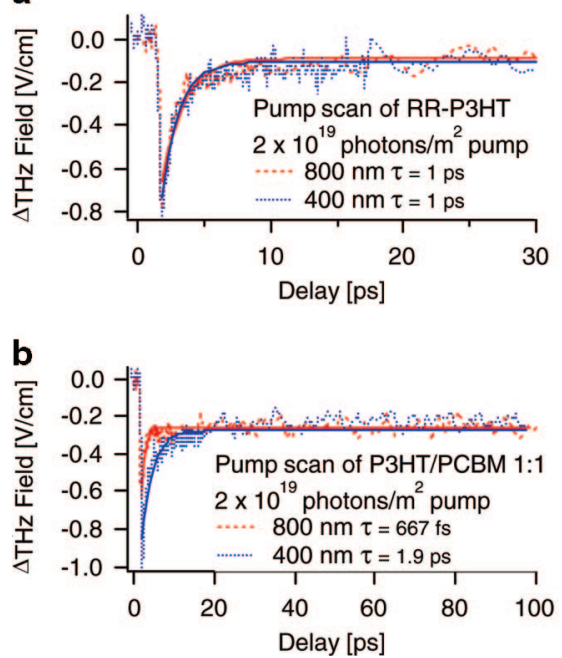

C
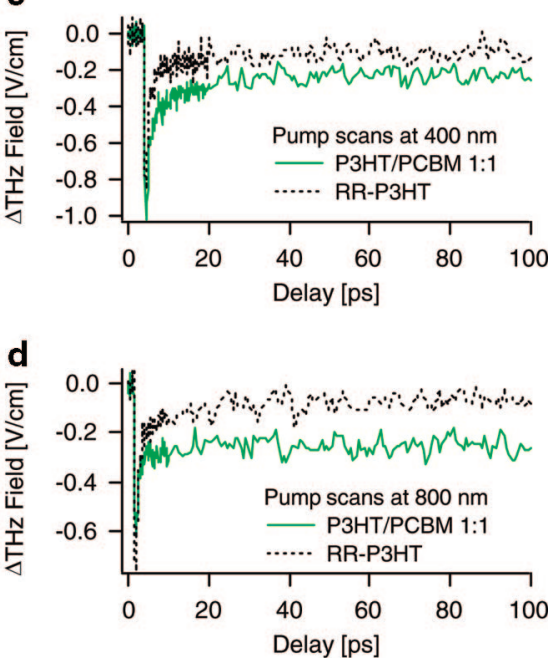

Figure 10. Comparison of photoconductivity dynamics in (a) RR-P3HT and (b) P3HT/PCBM 1:1 for 400 and 800 nm pumps, and at (c) 400 nm and (d) $800 \mathrm{~nm}$ excitation for RR-P3HT and P3HT/PCBM 1:1. Solid lines are stretched exponential fits with time constant $\tau$.

carriers are not the primary photoexcitation as they are clearly the minority product of absorption. ${ }^{19,26,27}$ We do not observe the polaron resonance at early delays reported by $\mathrm{Ai}$ et al. ${ }^{21}$ and instead conclude that bound excitons precede free-carriers as the primary photoproduct. The lack of an excitonic signature may mean the exciton polarizability is much smaller than the high-mobility free carrier response. As we observe linear fluence dependence (Figure 2), we can rule out second-order effects as the cause of free carrier generation via exciton dissociation. Though weak temperature dependence is characteristic of hot excitons, the presence of similar dynamics at $1.55 \mathrm{eV}$ excitation below the band edge does not agree with a hot exciton dissociation ${ }^{56}$ picture, which predicts an increase in free carrier yield with excitation energy. The two-dimensional lamellar structure, caused by efficient $\pi$-stacking, may aid in separating the initially photoexcited exciton, via interchain transfer, ${ }^{27}$ into free electrons and holes on separate chains. ${ }^{21}$ This picture is consistent with the low free carrier yield observed in isolated chains of MEH-PPV 27 in solution and the near unity yields in well-organized systems such as pentacene crystals. ${ }^{31}$ Future experiments comparing free carrier yields of isolated chains in solution and solid films of P3HT may be necessary to distinguish between these two cases. The large error in the estimated DC mobility is dictated exclusively by the error in the fit parameter $c$, which was $\leq 0.02$ in all cases and typically $\sim 0.01$. At long delays, the mobility was held fixed, which assumes that the mobility does not change significantly with delay. ${ }^{21}$ The most sensitive fit parameter in the DS model is the scattering time $\tau$. Unfortunately, the mobilities reported here correspond to scattering times with associated frequencies outside of our experimental bandwidth. The peak of the real and imaginary conductivities, for $c \sim-1$, correspond to the frequencies $(2 \pi \tau)^{-1}$ and $(3)^{1 / 3}(2 \pi \tau)^{-1}$ respectively. For a frequency range out to 2 $\mathrm{THz}$, the peak in the frequency-dependent real and imaginary conductivity can only be seen for scattering times greater than 80 and $137 \mathrm{fs}$, respectively. Silicon on sapphire, nanocrystalline silicon, ${ }^{57}$ and low-temperature-grown $\mathrm{GaAs}^{58}$ have all previously been reported to have scattering times lower than these values. Bandwidth out to $10 \mathrm{THz}$ would correspond to scattering times as low as $16 \mathrm{fs}$. If a larger frequency range was available, as seen through the use of polymer THz emitters and sensors, ${ }^{59,60}$ spectral features due to these characteristic times may be present and aid in the precise determination of these material properties.
The photoconductivity of 1:1 P3HT/PCBM (Figure 9) has the same trend as films of pure P3HT (Figure 8), showing a decaying real component with increased delay. The suppressed real conductivity is unexpected for efficient charge transfer of electrons from P3HT to PCBM, as a suppressed real component of the conductivity and negative linear imaginary component has previously been dubbed an excitonic signature. ${ }^{19}$ However, this trend was seen in previous reports, ${ }^{21}$ where it was attributed to the increasingly significant contribution from excitons as compared to free carriers as the carrier density decreases with increased delay. These authors attributed the change in the shape of the frequency-dependent complex conductivity to an excitonic component. However, inclusion of an excitonic fitting term of the form $\sigma_{2}(\omega)=-\varepsilon_{0} \omega \Delta \varepsilon$, where $\Delta \varepsilon$ is the change in the dielectric constant due to the presence of bound excitons, does not improve our fits. We see a decrease in the mobility of the bulk heterojunction (Table 2) versus the pure P3HT film when exciting at $400 \mathrm{~nm}$ (Table 1). This is unexpected, as PCBM improves the performance of P3HT-based devices. ${ }^{10}$ However, the presence of PCBM will decrease the ordering of the P3HT molecules, reducing $\pi$ stacking and the carrier mobility, making annealing, ${ }^{11-13,61}$ which was not performed here, often necessary to improve film morphology. We do see a marked increase in the photon-to-carrier yield, as expected, since PCBM aids in the creation of free charges by presenting an additional mechanism for exciton dissociation. ${ }^{19,21}$ It is surprising that the mobility found in the bulk heterojunction excited at $800 \mathrm{~nm}$ is larger than at $400 \mathrm{~nm}$, very near the value found in the pure P3HT film. Perhaps this is due to the selective excitation of P3HT over PCBM at $800 \mathrm{~nm}$, whereas both molecules absorb at $400 \mathrm{~nm}$. If we think of the extracted mobility as a weighted sum of those from the two components of the bulk heterojunction, then low electron mobility in PCBM, due to activated hopping between fullerenes, may account for the decreased effective mobility. However, this view would be inconsistent with OFET measurements that show the electron mobility in PCBM to be similar to the hole mobility in P3HT. ${ }^{15}$

Figure 10 compares the pump scans performed on RR-P3HT and a bulk heterojunction of 1:1 P3HT/PCBM at 400 and 800 $\mathrm{nm}$ excitation keeping the fluence the same. For P3HT, the two different pump wavelengths yield identical results (Figure 10a), within the error of our measurement. This result combined with the similar mobilities extracted at these two pump wavelengths 
(Table 1) indicates that both the high and low energy excitations may lead to the same carrier species over subpicosecond time scales. It has been suggested that singlet excitations populate the long-lived triplet state through intersystem crossing (ISC) in polythiophenes. ${ }^{41}$ Our results at these two excitation wavelengths could lend further support to this idea. The triplet state may be populated via ISC from the singlet state pumped at 400 $\mathrm{nm}$ and may also be directly excited via the weak absorption at $800 \mathrm{~nm}$. However, ISC in P3HT may not occur fast enough to explain our picosecond-scale results, as ISC typically occurs on 100 ps time scales. If ISC is significant, then the long-lived state that precedes electron transfer from P3HT may be 0.45 eV lower than the accepted lowest unoccupied molecular orbital (LUMO), which would be of importance to the OPV community in the future design of bulk heterojunctions. However, our results cannot definitively identify the lower energy absorption as a triplet state. Future UV/visible absorption studies of P3HT doped with heavier atoms, providing stronger spin-orbit coupling, that may increase this absorption feature would be necessary.

In the bulk heterojunction, the $800 \mathrm{~nm}$ pump results in a smaller photoresponse with faster decay. The decreased photoresponse is likely due to the lack of any absorption at 800 $\mathrm{nm}$ on the part of the PCBM. The $400 \mathrm{~nm}$ light provides more energy to the excited species, which may lengthen the cooling and electron transfer process.

Films of pure P3HT exhibit a larger initial photoresponse than the heterojunction at $800 \mathrm{~nm}$ excitation (Figure 10d). For the $400 \mathrm{~nm}$ pump, the heterojunction has a larger initial photoresponse (Figure 10c). These observations are likely due to the absorption properties of PCBM. For both excitation wavelengths, we see an increase in the long-lived photoresponse in the bulk heterojunction as compared to the pure P3HT film. As the LUMO of PCBM is thought to be 0.85 to $1.25 \mathrm{eV}$ lower than the accepted LUMO of P3HT, ${ }^{5,16}$ the electron transfer is still efficient for the lower energy $800 \mathrm{~nm}$ excitation.

\section{Summary}

We have used OPTP to examine the photoinduced charge generation and picosecond dynamics of P3HT and the bulk heterojunction P3HT/PCBM 1:1 with subicosecond resolution for excitation both above and below the energy gap for P3HT. We examined the temperature dependence of the photoinduced change in transmission and extracted the full complex frequencydependent conductivity, which was analyzed with the DS model to extract photon-to-carrier yields and carrier mobilities. We found similar conduction dynamics and mobilities for both excitation energies in pure $\mathrm{P} 3 \mathrm{HT}$, indicating the same fate for low and high-energy excitations. Further experiments are needed to determine whether the low energy absorption is a triplet state typically populated via ISC during higher energy excitation. Inclusion of PCBM was found to increase the carrier yield, but not the mobility, possibly because of disruption of ordering in the P3HT chains. Future broad bandwidth $(10 \mathrm{THz})$ terahertz studies may provide further insight by more accurately determining material constants through the identification of resonant spectral features.

Note Added in Proof. Recent density functional theory calculations (Northrup, J. E. Phys. Rev. B 2007, 76, 245202) have shown a room temperature mobility of $31 \mathrm{~cm}^{2} / \mathrm{Vs}$ in the $\pi$-stacking direction of perfectly crystalline P3HT. This is in reasonable agreement with the extracted mobilities reported here.

Acknowledgment. The authors thank Xuemei Zheng for helpful and stimulating conversations as well as $\mathrm{Xia} \mathrm{Li}$ and
Roman Sobolewski of Rochester Institute of Technology for their aid in the low-temperature work reported here. This material is based upon work supported by the STC program of the National Science Foundation, No. DMR 0120967.

\section{References and Notes}

(1) Surin, M.; Leclere, P.; Lazzaroni, R.; Yuen, J. D.; Wang, G.; Moses, D.; Heeger, A. J.; Cho, S.; Lee, K. J. Appl. Phys. 2006, 100, 033712.

(2) Wang, G.; Swensen, J.; Moses, D.; Heeger, A. J. J. Appl. Phys. 2003, 93, 6137.

(3) Shrotriya, V.; Ouyang, J.; Tseng, R. J.; Li, G.; Yang, Y. Chem. Phys. Lett. 2005, 411, 138.

(4) Dicker, G.; de Haas, M. P.; Siebbeles, L. D. A.; Warman, J. M. Phys. Rev. B 2004, 70, 045203.

(5) Chirvase, D.; Chiguvare, Z.; Knipper, M.; Parisi, J.; Dyakonov, V.; Hummelen, J. C. Synth. Met. 2003, 138, 299.

(6) Lioudakis, E.; Othonos, A.; Alexandrou, I.; Hayashi, Y. Appl. Phys. Lett. 2007, 91, 111117.

(7) Kim, J. Y.; Lee, K.; Coates, N. E.; Moses, D.; Nguyen, T.-Q.; Dante, M.; Heeger, A. J. Science 2007, 137, 222.

(8) Ma, W.; Kim, J. Y.; Lee, K.; Heeger, A. J. Macromol. Rapid Commun. 2007, 28, 1776

(9) Kim, Y.; Choulis, S. A.; Nelson, J.; Bradley, D. D. C.; Cook, S.; Durrant, J. R. J. Mater. Sci. 2005, 40, 1371.

(10) Huang, J.; Li, G.; Yang, Y. Appl. Phys. Lett. 2005, 87, 112105.

(11) Reyes-Reyes, M.; Kim, K.; Carroll, D. L. Appl. Phys. Lett. 2005, 87, 083506 .

(12) Kim, Y.; Choulis, S. A.; Nelson, J.; Bradley, D. D. C.; Cook, S.; Durrant, J. R. Appl. Phys. Lett. 2005, 86, 063502.

(13) Kim, Y.; Cook, S.; Tuladhar, S. M.; Choulis, S. A.; Nelson, J.; Durrant, J. R.; Bradley, D. D. C.; Giles, M.; Mcculloch, I.; Ha, C.; Ree, M. Nat. Mater. 2006, 5, 197.

(14) Yang, H.; LeFevre, S. W.; Ryu, C. Y.; Bao, Z. Appl. Phys. Lett. 2007, 90, 172116 .

(15) Li, G.; Shrotriya, V.; Huang, J.; Yao, Y.; Moriarty, T.; Emery, K.; Yang, Y Nat. Mater. 2005, 4, 864.

(16) Mihailetchi, V. D.; Xie, H.; de Boer, B.; Popescu, L. M.; Hummelen, J. C.; Blom, P. W. M.; Koster, L. J. A. Appl. Phys. Lett. 2006, $89,012107$.

(17) Manaka, T.; Lim, E.; Tamura, R.; Iwamoto, M. Nat. Photonics 2007, 1,581 .

(18) Prins, P.; Grozema, F. C.; Schins, J. M.; Savenije, T. J.; Patil, S.; Scherf, U.; Siebbeles, L. D. A. Phys. Rev. B 2006, 73, 045204.

(19) Hendry, E.; Schins, J. M.; Candeias, L. P.; Siebbeles, L. D. A.; Bonn, M. Phys. Rev. Lett. 2004, 92, 196601.

(20) Dicker, G.; de Haas, M. P.; Warman, J. M.; de Leeuw, D. M.; Siebbeles, L. D. A. J. Phys. Chem. B 2004, 108, 17818.

(21) Ai, X.; Beard, M. C.; Knutsen, K. P.; Shaheen, S. E.; Rumbles, G.; Ellingson, R. J. J. Phys. Chem. B 2006, 110, 25462.

(22) Hoofman, R. J. O. M.; de Haas, M. P.; Siebbeles, L. D. A.; Warman, J. M. Nature 1998, 392, 54.

(23) Warman, J. M.; Gelinck, G. H.; de Haas, M. P. J. Phys.: Condens. Matter 2002, 14, 9935.

(24) Beard, M. C.; Turner, G. M.; Schmuttenmaer, C. A. J. Phys. Chem. B 2002, 106, 7146.

(25) van Exter, M.; Grischkowsky, D. Appl. Phys. Lett. 1990, 56, 1694.

(26) Hendry, E.; Koeberg, M.; Schins, J. M.; Siebbeles, L. D. A.; Bonn,

M. Chem. Phys. Lett. 2006, 432, 441.

(27) Hendry, E.; Koeberg, M.; Schins, J. M.; Nienhuys, H. K.; Sundstrom, V.; Siebbeles, L. D. A.; Bonn, M. Phys. Rev. B 2005, 71, 125201.

(28) Thorsmølle, V.; Averitt, R.; Chi, X.; Hilton, D.; Smith, D.; Ramirez, A.; Taylor, A. J. Appl. Phys. Lett. 2004, 84, 891.

(29) Ostroverkhova, O.; Shcherbyna, S.; Cooke, D. G.; Egerton, R. F.; Hegmann, F. A.; Tykwinski, R. R.; Parkin, S. R.; Anthony, J. E. J. Appl. Phys. 2005, 98, 033701.

(30) Hegmann, F. A.; Tykwinski, R. R.; Lui, K. P. H.; Bullock, J. E.; Anthony, J. E. Phys. Rev. Lett. 2002, 89, 227403.

(31) Ostroverkhova, O.; Cooke, D. G.; Shcherbyna, S.; Egerton, R.; Hegmann, F. A.; Tykwinski, R. R.; Anthony, J. E. Phys. Rev. B 2005, 71, 035204.

(32) Ostroverkhova, O.; Cooke, D. G.; Hegmann, F. A.; Anthony, J. E.; Podzorov, V.; Gershenson, M. E.; Jurchescu, O. D.; Palstra, T. T. M. Appl. Phys. Lett. 2006, 88, 162101.

(33) Koeberg, M.; Hendry, E.; Schins, J. M.; van Laarhoven, H. A.; Flipse, C. F. J.; Reimann, K.; Woerner, M.; Elsaesser, T.; Bonn, M. Phys. Rev. B 2007, 75, 195216.

(34) Grozema, F. C.; van Duijnen, P. T.; Berlin, Y. A.; Ratner, M. A.; Siebbeles, L. D. A. J. Phys. Chem. B 2002, 106, 7791.

(35) Beard, M. C.; Turner, G. M.; Schmuttenmaer, C. A. Phys. Rev. B 2000, 62, 15764 
(36) Chen, Q.; Tani, M.; Jiang, Z.; Zhang, X. C. J. Opt. Soc. Am. B 2001, 18, 823 .

(37) Wu, Q.; Zhang, X.-C. Appl. Phys. Lett. 1995, 67, 3523.

(38) Kindt, J. T.; Schmuttenmaer, C. A. J. Chem. Phys. 1999, 110, 8589.

(39) Nienhuys, H. K.; Sundstrom, V. Phys. Rev. B 2005, 71, 235110

(40) Brown, P. J.; Thomas, D. S.; Kohler, A.; Wilson, J. S.; Kim, J.; Ramsdale, C. M.; Sirringhaus, H.; Friend, R. H. Phys. Rev. B 2003, 67, 064203.

(41) Sakurai, K.; Tachibana, H.; Shiga, N.; Terakura, C.; Matsumoto, M.; Tokura, Y. Phys. Rev. B 1997, 56, 9552.

(42) Silva, C.; Dhoot, A. S.; Russell, D. M.; Stevens, M. A.; Arias, A. C.; MacKenzie, J. D.; Greenham, N. C.; Friend, R. H. Phys. Rev. B 2001, 64, 125211.

(43) Planken, P. C. M.; Nienhuys, H.-K.; Bakker, H. J.; Wenckebach, T. J. Opt. Soc. Am. B 2001, 18, 313.

(44) Yu, G.; Phillips, S. D.; Tomozawa, H.; Heeger, A. J. Phys. Rev. B 1990, 42, 3004.

(45) Hannewald, K.; Bobbert, P. A. Appl. Phys. Lett. 2004, 85, 1535. (46) Papathanassiou, A. N.; Sakellis, I.; Grammatikakis, J. Appl. Phys. Lett. 2007, 91, 122911.

(47) Dyre, J. C.; Schroder, T. B. Rev. Mod. Phys. 2000, 72, 873.

(48) Smith, N. V. Phys. Rev. B 2001, 64, 155106.

(49) Walther, M.; Cooke, D. G.; Sherstan, C.; Hajar, M.; Freeman, M. R.; Hegmann, F. A. Phys. Rev. B 2007, 76, 125408.
(50) Jeon, T. I.; Grischkowsky, D.; Mukherjee, A. K.; Menon, R. Appl. Phys. Lett. 2001, 79, 4142.

(51) Moule, A. J.; Meerholz, K. Appl. Phys. Lett. 2007, 91, 061901.

(52) Dicker, G.; de Haas, M. P.; Siebbeles, L. D. A. Phys. Rev. B 2005, $71,155204$.

(53) Prins, P.; Grozema, F. C.; Siebbeles, L. D. A. J. Phys. Chem. B 2006, 110, 14659.

(54) Cooke, D. G.; Hegmann, F. A.; Young, E. C.; Tiedje, T. Appl. Phys. Lett. 2006, 89, 122103.

(55) Reedijk, J. A.; Martens, H. C. F.; B, B. H.; Michels, M. A. J. Phys. Rev. Lett. 1999, 83, 3904.

(56) Arkhipov, V. I.; Emelianova, E. V.; Bassler, H. Phys. Rev. Lett. 1999, $82,1321$.

(57) Cooke, D. G.; MacDonald, A. N.; Hryciw, A.; Wang, J.; Li, Q.; Meldrum, A.; Hegmann, F. A. Phys. Rev. B 2006, 73, 193311.

(58) Beard, M. C.; Turner, G. M.; Schmuttenmaer, C. A. J. Appl. Phys. 2001, 90, 5915.

(59) Zheng, X.; Sinyukov, A.; Hayden, L. M. Appl. Phys. Lett. 2005, 87,081115 .

(60) Zheng, X.; McLaughlin, C. V.; Cunningham, P. D.; Hayden, L. M. J. Nanoelect. Optoelect. 2007, 2, 58.

(61) Al-lbrahim, M.; Ambacher, O.; Sensfuss, S.; Gobsch, G. Appl. Phys. Lett. 2005, 86, 201120.

JP711827G 